\title{
PREDICTION OF SEIZURES IN ASPHYXIATED NEONATES
}

Early continuous video electroencephalography was used to detect seizures in term infants at high risk of hypoxic ischemic encephalopathy (HIE) in a study at University College Cork, and St Vincent's University Hospital, Dublin, Ireland. The predictive value of high-risk markers, the so-called " 3 -strikes" (Apgar score of $<5$ at $5 \mathrm{~min}, \mathrm{pH}<7.00$, and need for intubation) for EEG seizures, abnormal background EEG activity, and Sarnat grade, based on clinical behavior at 24 hours, was examined. Electrographic seizures occurred in 11 $(22 \%)$ of 49 infants suitable for analysis. Encephalopathy scored by Sarnat grades was severe in 6 infants, moderate in 18, and mild in 25. EEG background abnormalities were severe in 4, moderate in 16, and mild or moderate in 25 . Positive predictive values for neonatal seizures based on the 3-strikes were $18 \%$ for an Apgar score of $<5$ at $5 \mathrm{~min}, 16 \%$ for a $\mathrm{pH}<7.00$, and $21 \%$ for a need for intubation. A positive predictive value of $25 \%$ and a negative value of $77 \%$ were obtained for the 3 strikes combined. Base deficit $(<-15 \mathrm{mEq} / \mathrm{L})$ or lactate $(>10$ $\mathrm{mmol} / \mathrm{L}$ ) values substituted for $\mathrm{pH}$ in the 3 -strikes model did not improve its predictive value. Apgar score $<5$, nucleated red cells, and base deficit showed some association with Sarnat grade. The $5 \mathrm{~min}$ Apgar was significantly associated with both Sarnat grade and EEG grade. The risk of HIE after perinatal asphyxia may be predicted by Sarnat scores at 24 hours of age, but the neonatal EEG soon after delivery offers an early indication of seizures and neurologic outcome. Metabolic acidosis, in particular lactic acidosis may identify infants at risk of HIE but does not predict the occurrence of seizures or grade of encephalopathy. (Murray DM, Ryan CA, Boylan GB, Fitzgerald AP, Connolly S. Prediction of seizures in asphyxiated neonates: Correlation with continuous video-electroencephalographic monitoring. Pediatrics July 2006;118:41-46). (Respond: C Anthony Ryan MD, Unified Maternity Services, Erinville Hospital, Western Rd, Cork, Ireland).

COMMENT. Early monitoring of continuous EEG recordings was the best indicator of risk of neonatal seizures and severity of encephalopathy following perinatal asphyxia in the above study. Previous EEG studies used intermittent recordings that overlooked the occurrence of subclinical seizures. The model described by Shah PS et al (Arch Pediatr Adolesc Med 2006;160:729-736) offers an additional prognostic indicator within 4 hours of birth, of value in selection of cases for trials of neuroprotective therapies. The potential of therapies such as selective head cooling with systemic hypothermia has emphasized the importance of accurate and early prediction of outcome of neonatal encephalopathy in the first 6 hours of life (Gluckman PD et al. Lancet 2005;365:663-670).

Bedside EEG measures in encephalopathic infants are found to correlate with severity of cerebral injury defined by MRI in a study using 2-channel bedside amplitude-integrated EEG, at Royal Children's Hospital, Melbourne, Australia. (Shah DK et al. Pediatrics July $2006 ; 118: 47-55)$. A minimum amplitude of $<4 \mathrm{mcV}$ gives a specificity of $75 \%$, and a minimum of $6 \mathrm{mcV}$ has a greater sensitivity, allowing the bedside monitor to be used as a screening test for diagnosis and treatment. The amplitude-integrated EEG is useful as a prognostic indicator up to 3 days after admission of an asphyxiated infant to the NICU, even during anticonvulsant therapy. In 40 term-born $\mathrm{HI}$ encephalopathic infants, the lower the EEG amplitude, the greater the degree of MRI abnormality. 